\title{
Causes and (in)Determinism
}

\author{
Tomasz Placek • Jacek Wawer • Leszek Wroński
}

Received: 3 February 2013/Accepted: 3 February 2013/Published online: 21 February 2013

(C) The Author(s) 2013. This article is published with open access at Springerlink.com

The five papers of the current issue originate from selected talks of a workshop Causes and Tenses: Formal Perspectives, held in Kraków (Poland) in September 2010. The aim of this event was to explore two relatively recent projects in formal philosophy: a rigorous analysis of a modal and tensed notion of (in)determinism and a study of common causation by means of probabilistic extendability. In contrast to the Laplacean (in)determinism framed in tenseless terms of laws of nature or models of a theory, the first project sets out the task of analyzing a notion of (in)determinism exhibited in claims like "It is not settled yet if there will be a sea battle tomorrow; however, by tomorrow evening it will have been settled whether or not there was the battle". The analysis draws on the insights of the PriorThomason tense logic as well as Kaplan's logic of indexicals. Beginning with Belnap's (1992) theory of branching space-times, another aim of the project is to relate tensed (in)determinism to spatiotemporal structures of current physics. The present issue begins with three papers on some aspects of this variety of (in)determinism.

In the first paper Thomas Müller reflects on the notion of possible history, which is employed in all schools emerging from the Prior-Thomason theory of branching time. A history plays a distinctive role in assessing the truth values of the sentences to be evaluated in a given model. Possible histories also offer a theoretical grip on the concept of (in)determinism. They are usually defined as set-theoretically maximal objects of a certain kind. Although the formal details depend on the particular theory, the common idea is to read this maximality as maximality with respect to modal consistency. This calls in turn for spelling out a criterion of modal consistency (historicity) which would accord with insights of physics. The existing criteria, identifying possible histories with maximal chains or maximal upward directed subsets of a base set, are, however, in conflict with some relativistic

T. Placek $(\bowtie) \cdot J$. Wawer $\cdot$ L. Wroński

Kraków, Poland

e-mail: Tomasz.Placek@uj.edu.pl 
spacetimes. There is also a tension between the notion of possible histories and pragmatics, as maximal courses of events do not seem to be tractable enough to be useful as parameters in truth value evaluation. The paper considers two alternatives to the received notions of history whose aim is to dispose with these disadvantages.

The paper by Jacek Wawer ties into the ongoing debate concerning the status of the Thin Red Line (TRL) theories, which are, loosely speaking, branching theories enriched with the notion of the "actual history". The author believes that TRL theory should be conceived as a mix of actualism with eternalism. He discusses the semantic impact of such a metaphysical view and formulates his novel TRL solution as a set of presemantic, semantic, and postsemantic assumptions. The author argues that the new theory does not fall prey to the usual objections and is philosophically well motivated. He also offers an argument for a novel and more natural definition of the actuality operator in the branching setting.

The last paper of this bundle (by Tomasz Placek, Nuel Belnap, and Kohei Kishida) raises the question of topological features of indeterminism and investigates it in the framework of the theory of branching space-times, whose axiomatic form permits some definite answers. The authors supplement the branching space-times theory by four additional postulates, which allow one to define light cones with typical properties required by spacetimes of physics. Their main result is that with respect to a natural topology, a BST history with definable light cones satisfies the Hausdorff property. In contrast, with respect to this topology, the Hausdorff property fails in a multi-history BST model (excluding some pathological models). These results aim to clarify the recent debate concerning the applicability of BST histories in physical contexts.

The second topic of this special issue, probabilistic causation analyzed by means of probabilistic extendability, is represented by two joint papers, by Zalán Gyenis and Miklós Rédei and by Leszek Wroński and Michał Marczyk. The history of the subject is fascinating. One of the concepts crucial for the field-the Markov condition-is frequently motivated by Reichenbach's Common Cause Principle, which intuitively says that any correlations between two factors such that one is not a cause of the other share a common cause. This idea was given a precise mathematical formulation by Hans Reichenbach in his 1956 book The Direction of Time. The principle was observed to be similar to a premise of Bell's theorem and has been widely discussed by philosophers of science. The papers by van Fraassen (1982) and Sober (1988) convinced many that the Principle was simply false.

But a new turn in the late 1990's brought an even stricter rigor to the field. First, models for discovering causal structure on the basis of statistical information were constructed (see e.g. Pearl 2000), in which (some version of) the common cause principle is derivable. Second, Hofer-Szabó et al. (1999) observed that the question whether the principle is tenable has a clear-cut mathematical aspect. They asked if a probability space containing a pair of correlated events with no common cause can be extended to a larger probability space, in which the replicas of those events have a common cause, with the concepts of extension and replicas of probabilistic events having a precise mathematical meaning. This question initiated a fruitful research program, with a clear link to the philosophy of physics, as the applicability of the principle to different areas of physics is inextricably bound to its validity in 
nonclassical probability spaces. The two papers mentioned above document the latest development of this research program. In their paper Zalán Gyenis and Miklós Rédei establish a link between nonatomicity of a probability space and the validity of Reichenbach's Principle inside that space; the link holds regardless of whether the space is classical or nonclassical. In contrast, the last paper, by Leszek Wroński and Michał Marczyk, deals mainly with finite classical probability spaces and shows that a natural extension of the Reichenbachian notion is enough to prove that Reichenbach's Principle is valid in a lot bigger class of spaces then previously thought.

The workshop was held at the Department of Philosophy of the Jagiellonian University in Kraków (Poland) on September 10-11, 2010 and gathered seven speakers and around thirty participants. The event was generously sponsored by the Department of Philosophy (JU), Copernicus Center for Interdisciplinary Research, and research grant 668/N-RNP-ESF/2010/0.

Open Access This article is distributed under the terms of the Creative Commons Attribution License which permits any use, distribution, and reproduction in any medium, provided the original author(s) and the source are credited.

\section{References}

Belnap, N. (1992). Branching space-time. Synthese, 92(3), 385-434.

Hofer-Szabó, G., Rédei, M., \& Szabó, L. E. (1999). On Reichenbach's common cause principle and Reichenbach's notion of common cause. The British Journal for the Philosophy of Science, 50(3), 377-399.

Pearl, J. (2000). Causality: Models, reasoning, and inference. Cambridge: Cambridge University Press. Reichenbach, H. (1956). The Direction of Time. Berkeley, Los Angeles, Oxford: University of California Press.

Sober, E. (1988). The principle of the common cause. In J. H. Fetzer (ed.), Probability and causality (pp. 211-228). Dordrecht: D. Reidel Publishing Company.

van Fraassen, B. C. (1982). The Charybdis of realism: Epistemological implications of Bell's inequality. Synthese, 52, 25-38. 